\title{
Analysis of Two kinds of Complexation Agents in Nickel-phosphorus Alloy Brushing to Together Effect
}

\author{
Jiancheng Wang ${ }^{a}$, Yufeng Zhang ${ }^{b}$, Jie Lei , Shuang Xian \\ Wuhan Mechanical Technology College, Wuhan, Hubei 430075 , China \\ awzxw@163.com, bshujian@163.com
}

Keywords: Complexation agents; brushing; deposition speed; bear to decay ability.

\begin{abstract}
The effect of lactic acid(C3H6O3 ) and citric acid(C6H8O7 - $\mathrm{H} 2 \mathrm{O})$ in Ni-P alloy brushing liquid to Ni-P Alloy Brushing depositing speed and bear to decay ability is studied. Test a certificate, depositing with the increment of the lactic acid content speed to lower after increasing first; With the increment of the citric acid content, the deposition speed lower. When lactic acid content must, when the citric acid content is 5 20 g/l, brushing deposition speed to change not and greatly. Add lactic acid and citric acid two kinds of nets to match in the meantime, the ability obvious exaltation brushes deposition speed of plate the layer and bears corrosion function.
\end{abstract}

\section{Introduction}

The Ni-P alloy brushing liquid is increasingly widespread as a new type of surface treatment technology in the field of mechanical maintenance which is amorphous with high corrosion resistance,wear resistance and high hardness, etc[1]. However, there are still many problems to be studied, including the performance of the plating bath, the maintenance and regeneration of the plating bath, the performance of the coating and the effect of industrial application. Complexing agent is the main additive in the plating bath, and plays an important role in the plating solution. The organic acids and their salts are the main organic acids in the plating solution. There are three main effects: (1) In acid plating solution to prevent the formation of nickel nickel phosphate precipitation, (2) As buffer to prevent the $\mathrm{PH}$ value too fast,(3) Reduce the content of free $\mathrm{Ni}^{2+}[2]$. The commonly used complexing agent in the Ni-P alloy brushing liquid is lactic acid and citric acid. In this paper, the effect of the contents of lactic acid and citric acid in the Ni-P alloy brushing liquid and two kinds of complexing agents on the process of electroless Ni P alloy plating were studied.

\section{Experiment method}

The composition of the Ni-P alloy brushing liquid. The brushing liquid: NiSO $4 \cdot 6 \mathrm{H} 2 \mathrm{O}(\mathrm{Nickel}$ Salt), $153 \mathrm{~g} / 1$; NaH2PO2·H2O(Sodium Hypophosphite), 63g/1; C3H6O3(Lactic Acid), 10 60g/l;C6H8O7•H2O(Citric Acid), 5 30g/1; additive, appropriate amount; PH,4.5(the brushing liquid prepared by pure reagent and distilled water); working voltage, $12-14 \mathrm{~V}$; brushing liquid temperature, room temperature.

The experimental materials and equipment. The sample using $240 \mathrm{~mm} \times 70 \mathrm{~mm} \times 2 \mathrm{~mm}$ Q235

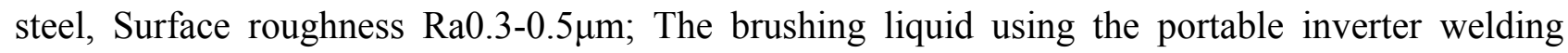
machine using charging plating developed by the Wuhan Ordnance Officer School.The deposition rate of the coating was determined by weighing method: $V=\frac{W_{1}-W_{2}}{s \cdot p \cdot t} \times 10^{4}$. In the formula: $v$ deposition $\operatorname{rate}(\mu \mathrm{m} / \mathrm{h})$; w1 - the sample weight after brush plating $(\mathrm{g})$; w2- the sample weight before brush plating $(\mathrm{g})$; S- sample brush plating area $(\mathrm{cm} 2) ; \rho$ - brush plating alloy density $(\mathrm{g} / \mathrm{cm} 3)$, the $\rho$ 
value is $7.8 \mathrm{~g} / \mathrm{cm} 3$; $\mathrm{t}$ - brush plating time(h).

\section{Experimental result and analysis}

The effects of lactic acid content on the deposition rate of the brush plating. The content of lactic acid in the brush plating has a great influence on the deposition rate of the brush plating, and the test results are shown in Figure 1.

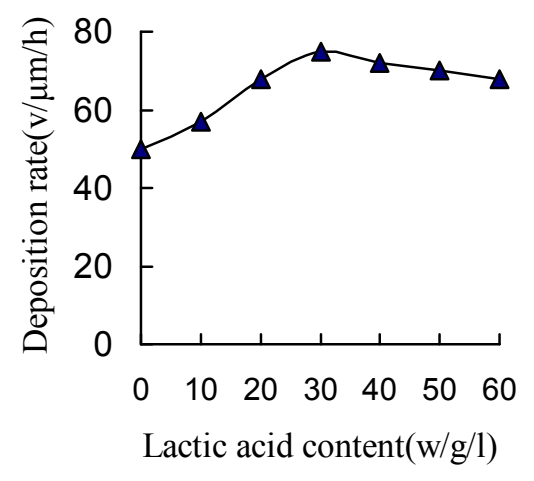

Figure 1 Relationship between the content of lactic acid and the deposition rate of the brush plating

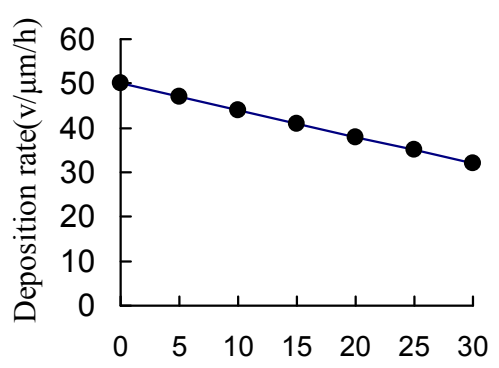

Figure 2 Relationship between the content of citric acid and the deposition rate of the brush plating

The results show that when the content of lactic acid in the brush plating solution is about $\leq 30 \mathrm{~g} / \mathrm{l}$, the deposition rate increases with the increase of the content of lactic acid, and the deposition rate of the coating increases with the increase of the content of lactic acid. This is because the organic additives can increase the deposition rate, because they are adsorbed on the surface of the plating, and improve the surface activity of the coating. The surface activity of the coating increases with the increase of the current efficiency of the brush plating process, which accelerates the reduction of the metal ions, and the addition of complexing agent can work stably with higher $\mathrm{pH}$ value, and make the $\mathrm{pH}$ value decrease slowly[3]. In the lactic acid content is about $\leq 30 \mathrm{~g} / \mathrm{l}$, strong ligand end reached the saturation point, with the increase of lactic acid, brush coating deposition rate was increased when reached saturation after brush deposition rate after a maximum value, when the ligand content increased more than brush deposition rate maxima corresponding to the ligand content, brush the deposition rate decreased. When the lactic acid content in the $30 \mathrm{~g} / \mathrm{l}$, the deposition rate of the brush plating was decreased, when the amount of was over $50 \mathrm{~g} / \mathrm{l}$, the deposition rate of the brush plating changed little, so the optimum value of lactic acid was good at $30 \mathrm{~g} / \mathrm{l}$.

The effects of citric acid on the deposition rate of the brush plating. The citric acid as the complexing agent has a greater impact on the deposition rate of the brush plating, the test results are shown in Figure 2.

The results show that when the content of citric acid in brush plating solution is $5 \sim 30 \mathrm{~g} / \mathrm{l}$, the deposition rate decreases with the increase of its content. The reason may be related to the spatial structure of the ligand, due to the coordination of citrate ion volume larger, nickel ions in and their ligands to form complex ions, and the size becomes larger, hindering the nickel ion[4] to be plated substrate having the ability to respond to the activity and thus adding a complexing agent citric acid salt brush deposition rate will be reduced accordingly. 
The effects of two kinds of chelating agents on the deposition rate and corrosion resistance of the brush plating. When the lactic acid content in the brush plating solution is $30 \mathrm{~g} / \mathrm{l}$, the change of the citric acid content and the deposition rate of the brush plating is shown in Figure 3.

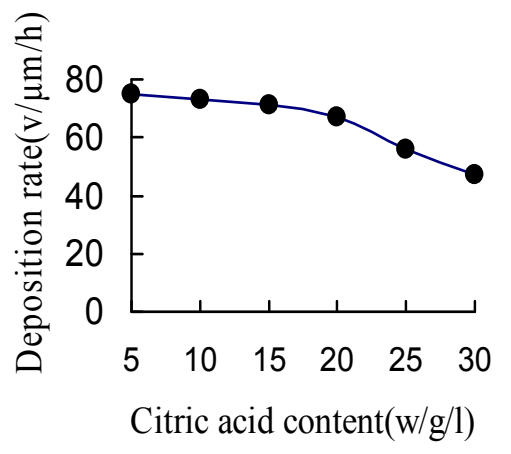

Figure 3 Relationship between the content of citric acid and the deposition rate of the brush plating on the content of lactic acid in $30 \mathrm{~g} / 1$

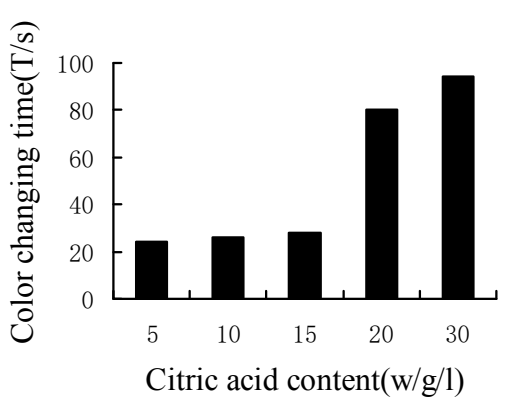

Figure 4 Relationship between the content of citric acid and the time of color change of brush plating

When the citric acid content was 5 20g/l, the deposition rate of the coating was not changed; When the citric acid content was over $20 \mathrm{~g} / 1$, the deposition rate of the brush plating decreased rapidly. However, the presence of citric acid can significantly improve the corrosion resistance of the brush plating, when the content of citric acid, the brush plating in $68 \%$ of the concentration of HNO3 in Figure 4.

By Figure 4, when the content of citric acid in brush plating solution is lower than $20 \mathrm{~g} / 1$, the average color change time of brush plating is less than $30 \mathrm{~s}$; Brush coating by citric acid content is not less than $20 \mathrm{~g} / 1$, color more than $80 \mathrm{~s}$. This is because when the content of citric acid is lower than $20 \mathrm{~g} / \mathrm{l}$, the structure of the brush plating layer is microcrystalline and amorphous, and the coating has many defects, so it has poor corrosion resistance; When the citric acid content is more than $20 \mathrm{~g} / 1$, the structure of the brush coating is amorphous [5], so it has good corrosion resistance. The results show that the deposition effect of the composite complexing agent is better than that of a single complexing agent.

\section{Conclusion}

(1) With the increase of lactic acid content in brush plating solution, the deposition rate first increased and then decreased, and the optimal value of lactic acid is $30 \mathrm{~g} / \mathrm{l}$; When the content of citric acid in brush plating solution is $5 \sim 30 \mathrm{~g} / 1$, the deposition rate decreases with the increase of its content.

(2) When the content of lactic acid is $30 \mathrm{~g} / 1$ and the content of citric acid is 5 20g/l in the brush plating solution, the deposition rate changed little; When the citric acid content is more than or equal to $20 \mathrm{~g} / \mathrm{l}$, the brush coating corrosion resistance improved significantly, therefore, the composite complexing agent brush coating deposition effect is better than using single complexing agent. 


\section{References}

[1] X.F. Xiao, R.F. Liu. Materials Protection, Vol. 36(2003) No.4, p.46-47. (In Chinese)

[2] C.Y. Ban, L.T. Zhang and L.J. Chen. Surface Technology, Vol. 28(1999) No.3, p.9-11. (In Chinese)

[3] D.L. Zhang, S.P. Gong and D.X. Zhou. Development and Application of Materials, Vol. 15 (2000) No.1, p.5-8. (In Chinese)

[4] ZJ. Zhu, P. Yu and F.H. Wang. Electroplating \& Finishing, Vol. 22(2003) No.5, p.22-25. (In Chinese)

[5] Z.X. Lv, C.F. Nie and N.H. Wang.Materials for Mechanical Engineering, Vol. 23(1999) No.3, p.3-5. (In Chinese) 